\title{
Stability and attractors for the quasi-steady equation of cellular flames
}

\author{
C.-M. BRAUNER ${ }^{\dagger}$ \\ Mathématiques Appliquées de Bordeaux, Université Bordeaux I, 33405 Talence Cedex, France \\ M. FRANKEL ${ }^{\dagger}$ \\ Department of Mathematical Sciences, Indiana University - Purdue University Indianapolis, \\ Indianapolis, IN 46202-3216, USA \\ J. HULSHOF ${ }^{\S}$ \\ Faculty of Sciences, Mathematics and Computer Science division, Vrije Universiteit Amsterdam, \\ 1081 HV Amsterdam, The Netherlands \\ AND \\ V. ROYTBURD ${ }^{\text {II }}$ \\ Department of Mathematical Sciences, Rensselaer Polytechnic Institute, \\ Troy, NY 12180-3590, USA
}

[Received 13 May 2005 and in revised form 15 May 2006]

\begin{abstract}
We continue the study of a simple integro-differential equation: the quasi-steady equation (QS) of flame front dynamics. This equation is dynamically similar to the Kuramoto-Sivashinsky (KS) equation. In [FGS03], where it was introduced, its well-posedness and proximity for finite time intervals to the KS equation in Sobolev spaces of periodic functions were established. Here we demonstrate that QS possesses a universal absorbing set, and a compact attractor. Furthermore we show that the attractor is of a finite Hausdorff dimension, and give an estimate on it. We discuss relationships with the Kuramoto-Sivashinsky and Burgers-Sivashinsky equations.
\end{abstract}

\section{Introduction}

In a recent paper [BFHS05] we studied the quasi-steady (QS) model of cellular flames:

$$
\Phi_{t}+\frac{1}{2} \Phi_{x}^{2}=(I-\alpha A) \Phi_{x x}
$$

where

$$
A:=\left(I-\partial_{x}^{2}\right)^{-1} .
$$

The equation in (1) arises as a certain truncation of a weakly-nonlinear version of a more detailed model of cellular flames (the $\kappa-\theta$ model) introduced in [FGS03]. The $\kappa-\theta$ model may serve as a basis for the description of the flame interaction with the background flow-field, which so far has

\footnotetext{
${ }^{\dagger}$ E-mail: brauner@math.u-bordeaux1.fr

‡E-mail: mfrankel@tsunami.math.iupui.edu

E-mail: jhulshof@few.vu.nl

EI E-mail: roytbv@rpi.edu
} 
been developed only for cellularly stable flames. As we argue below, the QS equation, in contrast with the Kuramoto-Sivashinsky equation, presents a uniform approximation of the spectrum of the original combustion problem.

Equation (1) was shown to be well-posed in Sobolev spaces of periodic functions. Moreover, for a fixed time interval in a certain sense solutions of (1) become uniformly close to those of the Kuramoto-Sivashinsky (KS) equation

$$
\Phi_{t}+\frac{1}{2} \Phi_{x}^{2}=(1-\alpha) \Phi_{x x}-\Phi_{x x x x}
$$

as the instability parameter $\varepsilon=1-\alpha$ approaches zero.

The latter result seems to imply a strong resemblance between the dynamics generated by the two equations, in particular one would expect that QS is capable of generating a cellular structure and chaotic behavior for an appropriate range of parameters. Indeed, some preliminary results of a direct numerical simulation [BFHS05] confirm this expectation. Thus, "quasi-steady" in the name of (1) relates rather to the way it was originally introduced, and is in no way indicative of its rich dynamics.

However, one wonders whether the dynamical features, such as stability/dissipativity and existence of a compact attractor of finite Hausdorff dimension that were established rigorously for $\mathrm{KS}$, can be demonstrated for the QS model. The positive answer to this question is the main result of the current paper.

Since the time of writing the paper [BFHS05] our view of the nature of (1) has somewhat evolved, therefore we present a slightly different discussion of its basic properties that are expressed through its dynamics. First, it is easy to show that (1) can be represented in a slightly different and more convenient form:

$$
\Phi_{t}+\frac{1}{2} \Phi_{x}^{2}=\Phi_{x x}+\alpha(I-A) \Phi
$$

This form is rather reminiscent of yet another dissipative system that has been a subject of discussion in recent years (see [RS87, G94, BKS01]), the so called Burgers-Sivashinsky (BS) equation:

$$
\Phi_{t}+\frac{1}{2} \Phi_{x}^{2}=\Phi_{x x}+(\alpha-1) \Phi
$$

The coefficient $\alpha-1$ (we assume $\alpha>1$ ) is chosen in such a form here simply to adjust the neutral stability wave number (at $\omega(k)=0$, see the dispersion relations below) to the other two equations for easier comparison.

All three of these equations (2), (3), and (4) share the same basic quality revealed by linear stability analyses, namely long-wave destabilization, which is suppressed by the dominant dissipative principal term for small wave lengths. The respective dispersion relations read

$$
\begin{aligned}
& \omega(k)=(\alpha-1) k^{2}-k^{4}, \\
& \omega(k)=-k^{2}+\frac{\alpha k^{2}}{1+k^{2}}, \\
& \omega(k)=-k^{2}+\alpha-1 .
\end{aligned}
$$

The neutral stability curve is the same for all three equations, it is the parabola

$$
k(\alpha)=\sqrt{\alpha-1}
$$


As one can easily see, in the long-wave part of the spectrum $k \ll 1$, the dispersion relation for QS is similar to that of $\mathrm{KS}$,

$$
\omega(k)=-k^{2}+\frac{\alpha k^{2}}{1+k^{2}}=(\alpha-1) k^{2}-\alpha k^{4}+\mathcal{O}\left(k^{6}\right)+\cdots,
$$

while for the short waves $k \gg 1$ its decay (in contrast to KS) $\omega \sim-k^{2}$ is identical to that of BS.

In order to understand which dispersion relation better reflects the physical reality we should turn to the original combustion problem. The real-life flames constitute a rather intricate physical system involving the fluid dynamics of the multicomponent gaseous mixture, the multistep chemical kinetics, as well as the molecular and radiative transfer. It transpires, however, that the cellular instability may be successfully captured by a simple reaction-diffusion system. In an appropriate range of physico-chemical parameters a subsequent reduction to a free interface problem can be performed [MS79]. The dispersion relation for this free-interface problem reads (see e.g. [BL00])

$$
\left(1+4 \omega+4 k^{2}-\alpha\right)\left(1-\sqrt{\left(1+4 \omega+4 k^{2}\right)}\right)-2 \alpha \omega=0 .
$$

To compare the latter dispersion relation with (KS) and (QS) we turn to the scaling employed in the derivation of the KS equation [Siv]: $\varepsilon:=\alpha-1=o(1), k^{2}=\mathcal{O}(\varepsilon)$, and $\omega=\mathcal{O}\left(\varepsilon^{2}\right)$. Then, up to $\varepsilon^{2},(\mathrm{FI})$ yields

$$
\omega=(\alpha-1) k^{2}-4 k^{4} .
$$

It is easy to see that for $k \gg 1,(\mathrm{FI})$ yields $\omega=-k^{2}$.

Thus, while in the long-wave approximation the behavior of the exact dispersion relation for the free-interface problem is mimicked by $\mathrm{KS}$ and QS, its decay at infinity is identical to BS and once again to QS. The three equations QS, KS and BS share the same nonlinearity, and the destabilization-dissipation mechanism is identical for all three (as we show below). The nonlinearity disperses the energy from the unstable modes to the rest of the spectrum sufficiently for the principal term to dissipate the energy excess.

Therefore, the (linear part of) QS equation presents a uniform approximation of the spectrum of the original combustion problem. Fig. 1 illustrates our remarks concerning the comparative linear stability analyses of the three equations on the one hand, and the free-interface problem on the other hand. Fig. 11(left) represents the dispersion relations while Fig. 11 (right) is a magnification of its long wave region. It is necessary to remark that while KS is the model equation for the onset of cellular-chaotic dynamics, one may expect that some important features of global dynamics of the original physical system should be better described by the QS equation, as they may be influenced by the manner in which the energy created by the long-wave instability is channeled to the rest of the spectrum.

It is necessary to remark that in Fig. 1 for comparison with the free boundary problem, the dispersion relation in (KS) had to be modified as follows:

$$
\omega(k)=(\alpha-1) k^{2}-4 k^{4} .
$$

Indeed, when the KS equation is derived from the free boundary problem the factor 4 arises naturally. Traditionally however, it is scaled out as in (KS). A similar factor would appear in the QS equation: the operator $\left(I-\partial_{x}^{2}\right)^{-1}$ should be replaced by $\left(I-4 \partial_{x}^{2}\right)^{-1}$. However when QS was introduced as an ad hoc model in [BFHS05] the factor was left out for comparison with the traditional KS. This factor is reintroduced in the dispersion relations depicted in Fig. 1. 

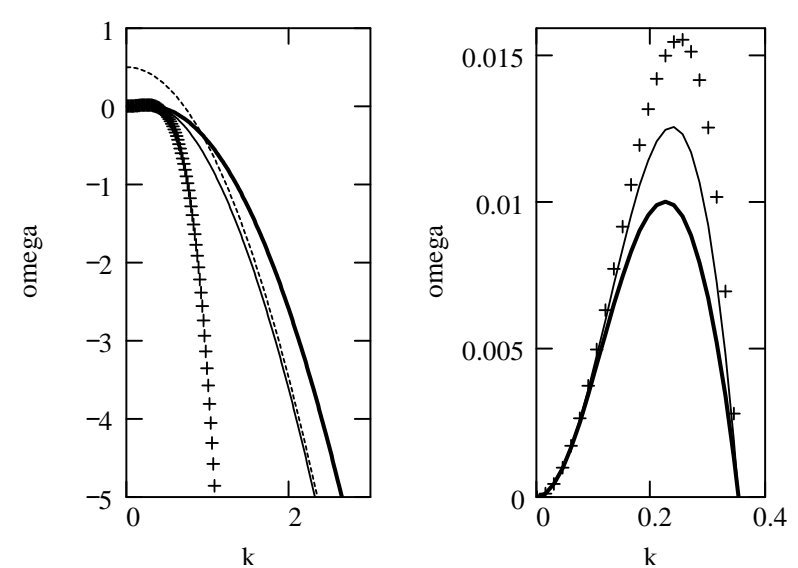

FIG. 1. Dispersion relations for the KS (pluses), the QS (thin line), the BS (dots) and the original free-interface problem (thick line) for $\alpha=3 / 2$. A magnification of the long wave region is on the right.

It appears that the QS equation represents as it were a middle ground between the KS and BS. Indeed, on the one hand the dynamics of the QS is qualitatively identical to the KS regarding the basic ability to generate chaotic cellular solutions. On the other hand, the standard energy type upper bounds on the size of the absorbing set and the dimension of the attractor are practically identical for both QS and BS.

It should be noted that, unlike KS and QS and in spite of the rather sizable upper bound for the attractor, the dynamics generated by the BS equation is asymptotically trivial (see e.g. [BKS01, G94]) as all its solutions gravitate to steady states dictated by the length of the interval and the parameter. Consequently, the true dimension of the attractor should be equal to zero. This is obviously due to the fact that, in contrast to the KS and QS equations, the most linearly unstable mode corresponds to the infinite wave length $k=0$.

If one carries out the estimates of the attractor for BS in the Sobolev spaces of $L$-periodic functions, one obtains an estimate for the Hausdorff dimension that is a certain power of the period $L$, with no indication whatsoever on the triviality of the dynamics on the attractor. In fact, the bounds for BS in [G94] are practically identical to the ones for QS, obtained in the present paper.

This is a rather disturbing outcome, especially in view of the considerable effort [NST85, G94, CEES93, GA04] to lower the bounds on the size of the absorbing set, and consequently the dimension of the attractor. This effort is based on the belief that these characteristics measure the "amount of chaos" or "the strength of turbulence" in the solutions of KS. Therefore one should keep in mind that the estimates of the Hausdorff dimension of the attractor are just upper bounds. Nonetheless, they should be considered as important characteristics of the infinite-dimensional dynamics. Thus, the main results of the paper, the estimates on the size of the absorbing set and on the Hausdorff dimension of the attractor for the QS equation, are of a considerable interest.

Technically, in the stability section the paper represents a combination of the methods employed in [G94] and [CEES93], while the estimate of the dimension follows rather faithfully the book of Temam [Temam]. In view of the above comments we did not attempt to obtain necessarily the lowest estimate opting for the transparency of presentation. We should remark that, as it seems, the method suggested in [GA04] can be customized for the QS, leading to a lower estimate for the absorbing ball, $R \sim L$. 
We preface the main part of the paper by a brief summary of results of [BFHS05] in Sec. 2. We conclude the introduction with a remark on the notation convention: unless otherwise indicated, all the spatial integrals are assumed to be over the spatial period, i.e., we always write $\int \ldots \mathrm{d} x$ instead of $\int_{-L / 2}^{L / 2} \ldots \mathrm{d} x$.

\section{Mathematical setting and previous results}

We consider (1) in the class of $L$-periodic functions in $x$. It is more convenient and rather conventional (cf. [Temam]) to study the equation differentiated with respect to $x$

$$
u_{t}+u u_{x}=u_{x x}+\alpha(I-A) u .
$$

Here $u=\Phi_{x}$ is in the class of periodic functions with zero average; $\Phi$ can be easily recovered from $u$. It is (7) that we study below. In fact the discussion in the introduction also concerns rather the differentiated forms of the three equations QS, KS and BS.

For integer or arbitrary real $s$ we denote by $H^{s}$ the usual Sobolev spaces of $L$-periodic (generalized) functions with zero average. We remark that $A$ is positive definite:

$$
\int u A u \geqslant 0,
$$

which is frequently used below.

The global existence is stated as follows:

THEOREM 2.1 ([BFHS05]) Let $u_{0} \in H^{s}$ where $s=0,1,2,3, \ldots$ Then (7p) has a unique solution on any time interval $[0, T]$ with $u(\cdot, 0)=u_{0}$. The solution belongs to $L^{2}\left(0, T ; H^{s+1}\right) \cap$ $C^{0}\left([0, T] ; H^{s}\right)$, and has $u_{t} \in L^{2}\left(0, T ; H^{s-1}\right)$, and the corresponding norms are bounded by a constant which depends only on $\alpha, s, L, T$ and $\|\Psi(0)\|_{s}$. Thus the solution is smooth for $t>0$.

In order to explain the asymptotic proximity result in [BFHS05] we note first that in contrast with (2) the usual form of KS in (11) below is parameter-free. It was derived from the original freeinterface problem via a rescaling of coordinates under the assumption $\varepsilon=\alpha-1 \ll 1$. For this reason, to compare the solutions of QS and KS, the QS should also undergo the same rescaling.

Thus, we are interested in $\alpha=1+\varepsilon$, where $\varepsilon$ is a (small) fixed positive number. We take the period to depend on $\varepsilon$, introducing a reference period $L_{0}>0$ and (reference) time interval [0, $\left.T_{0}\right]$. The reference space and time variables are denoted by $\xi$ and $\tau$ :

$$
x=\xi / \sqrt{\varepsilon}, \quad t=\tau / \varepsilon^{2}, \quad u=\varepsilon \varphi .
$$

We consider $\sqrt{1}$ in the class of periodic functions with period $L_{0} / \sqrt{\varepsilon}$ in $x$, on the time interval $\left[0, T_{0} / \varepsilon^{2}\right]$ in $t$. The rescaled version of $(1)$ is

$$
\left(I-\varepsilon \partial_{\xi}^{2}\right)\left(\varphi_{\tau}+\frac{1}{2} \varphi_{\xi}^{2}\right)+\varphi_{\xi \xi \xi \xi}+\varphi_{\xi \xi}=0 .
$$

The solution of (10) is sought as $\varphi=U+\varepsilon w$, so that for $\varepsilon=0$ one recovers the (parameter-free) KS:

$$
U_{\tau}+\frac{1}{2} U_{\xi}^{2}+U_{\xi \xi \xi \xi}+U_{\xi \xi}=0 .
$$


The equation for the perturbation $w$ reads

$$
\left(I-\varepsilon \partial_{\xi}^{2}\right)\left[w_{\tau}+\frac{1}{2}\left(2 U_{\xi} w_{\xi}+\varepsilon w_{\xi}^{2}\right)+w_{\xi \xi \xi \xi}+w_{\xi \xi}\right]=\partial_{\xi}^{2}\left(U_{\tau}+\frac{1}{2} U_{\xi}^{2}\right)
$$

We assume for simplicity that both QS and KS start from exactly the same initial condition, i.e. $w(\xi, 0)=0$. Then one can perform an energy type estimate for $w$ to obtain

THEOREM 2.2 Let $U_{0} \in H^{3}$. There exists $\varepsilon_{0}>0$ such that, whenever $0<\varepsilon<\varepsilon_{0},|w(\xi, \tau)| \leqslant C$ for all $(\xi, \tau)$ in $\left[-L_{0} / 2, L_{0} / 2\right] \times\left[0, T_{0}\right]$. The number $\varepsilon_{0}$ and the bound $C$ depend only on $U_{0} \in H^{3}$ and $T_{0}$.

For the original problem, $\Phi=\varepsilon \varphi, \varphi=U+\varepsilon w$, the theorem yields a nearness estimate:

COROLLARY 2.3

$$
\max \left|\Phi(x, t)-\varepsilon U\left(x \sqrt{\varepsilon}, t \varepsilon^{2}\right)\right| \leqslant C \varepsilon^{2}
$$

for $|x| \leqslant L_{0} /(2 \sqrt{\varepsilon}), 0 \leqslant t \leqslant T_{0} / \varepsilon^{2}$.

\section{Absorbing set}

The essence of the dissipative nature of QS is expressed in uniform boundedness of solutions. The well-posedness results of [BFHS05] do not provide a necessary bound. The purpose of this section is to establish such a bound.

In the spirit of [G94, Proposition 1] we need the following result:

LEMMA 3.1 Define

$$
b(x)= \begin{cases}0, & |x|>\varepsilon, \\ \frac{B}{\varepsilon}(1-|x| / \varepsilon), & |x| \leqslant \varepsilon\end{cases}
$$

(i.e., the graph of $b$ is a triangle of area $B$ with base $2 \varepsilon$ ). Then for any $u \in H^{1}$,

$$
\int b(x) u(x)^{2} \mathrm{~d} x \leqslant \frac{4}{B}\langle u b\rangle^{2}+2 B \varepsilon \int u^{\prime}(x)^{2} \mathrm{~d} x
$$

where

$$
\langle u b\rangle=\int b(y) u(y) \mathrm{d} y .
$$

Proof. On the interval $x, y \in(-\varepsilon, \varepsilon)$,

$$
u(x)=u(y)+\int_{y}^{x} u^{\prime}(z) \mathrm{d} z
$$

We multiply this equation by $b(y)$ and integrate with respect to $y$ to obtain

$$
\begin{aligned}
B u(x) & =\langle u b\rangle+\int_{-\varepsilon}^{\varepsilon} b(y) \mathrm{d} y \int_{y}^{x} u^{\prime}(z) \mathrm{d} z \\
& =:\langle u b\rangle+\int_{-\varepsilon}^{\varepsilon} K(x, z) u^{\prime}(z) \mathrm{d} z=:\langle u b\rangle+\hat{K} u^{\prime}
\end{aligned}
$$


where $\hat{K}$ is an integral operator on $L^{2}[-\varepsilon, \varepsilon]$ with the kernel

$$
K(x, z)= \begin{cases}\int_{z}^{\varepsilon} b(y) \mathrm{d} y & \text { if } z \geqslant x, \\ \int_{-\varepsilon}^{z} b(y) \mathrm{d} y & \text { if } z \leqslant x .\end{cases}
$$

Next we estimate the $L^{2}$ norm of $u$ on $(-\varepsilon, \varepsilon)$ :

$$
\begin{aligned}
\int_{-\varepsilon}^{\varepsilon} u(x)^{2} \mathrm{~d} x & =\frac{1}{B^{2}} \int_{-\varepsilon}^{\varepsilon}\left[\langle u b\rangle^{2}+2\langle u b\rangle\left(\hat{K} u^{\prime}\right)(x)+\left(\hat{K} u^{\prime}\right)(x)^{2}\right] \mathrm{d} x \\
& \leqslant \frac{2}{B^{2}} \int_{-\varepsilon}^{\varepsilon}\left[\langle u b\rangle^{2}+\left(\hat{K} u^{\prime}\right)(x)^{2}\right] \mathrm{d} x=\frac{2}{B^{2}}\left[2 \varepsilon\langle u b\rangle^{2}+\int_{-\varepsilon}^{\varepsilon}\left(\hat{K} u^{\prime}\right)(x)^{2} \mathrm{~d} x\right] \\
& \leqslant \frac{4 \varepsilon}{B^{2}}\langle u b\rangle^{2}+\frac{2}{B^{2}}\|\hat{K}\|^{2} \int_{-\varepsilon}^{\varepsilon}\left(u^{\prime}\right)^{2}(x) \mathrm{d} x .
\end{aligned}
$$

It is clear that $|K(x, z)| \leqslant B$, hence the Hilbert-Schmidt norm satisfies

$$
\|\hat{K}\|^{2}=\int_{-\varepsilon}^{\varepsilon} \int_{-\varepsilon}^{\varepsilon}|K(x, z)|^{2} \mathrm{~d} x \mathrm{~d} z \leqslant B^{2} \varepsilon^{2},
$$

Therefore

$$
\begin{aligned}
\int b(x) u^{2}(x) \mathrm{d} x & \leqslant \sup b \int_{-\varepsilon}^{\varepsilon} u(x)^{2} \mathrm{~d} x \\
& \leqslant \frac{B}{\varepsilon}\left[\frac{4 \varepsilon}{B^{2}}\langle u b\rangle^{2}+\frac{2}{B^{2}}\|\hat{K}\|^{2} \int_{-\varepsilon}^{\varepsilon}\left(u^{\prime}\right)(x)^{2} \mathrm{~d} x\right]=\frac{4}{B}\langle u b\rangle^{2}+2 B \varepsilon \int u^{\prime}(x)^{2} \mathrm{~d} x .
\end{aligned}
$$

The selection of $\varepsilon=1 /(8 B)$ in $(13)$ gives rise to the following important inequality:

COROLLARY 3.2 (Poincaré type inequality)

$$
\int b(x) u(x)^{2} \mathrm{~d} x \leqslant \frac{4}{B}\langle u b\rangle^{2}+\frac{1}{4} \int u^{\prime}(x)^{2} \mathrm{~d} x .
$$

The main result of this section is the following

THEOREM 3.3 (Existence of an absorbing ball) Let $L \geqslant 1 /(2 \alpha)$. Then for any solution $u$ of (7),

$$
\limsup _{t \rightarrow \infty}\|u(\cdot, t)\| \leqslant C_{a} \alpha L^{3 / 2},
$$

where $C_{a}$ is a universal constant; $\|\cdot\|$ is the $H^{0}$-norm.

REMARK 3.4 The assumption $L>1 /(2 \alpha)$ covers the most interesting case, when unstable modes exist. Indeed, the smallest eigenvalue for the $L$-periodic problem with zero mean is $\lambda=(2 \pi / L)^{2}$. From the neutral stability curve we get $L>2 \pi / \sqrt{\alpha-1}$ for the unstable modes to appear, which is a stronger condition than $L>1 /(2 \alpha)$. It is a rather simple matter to prove that all solutions decay exponentially in time in the opposite case. 
Proof of Theorem 3.3 Let $s$ be a fixed periodic function with zero mean (to be selected later). Introduce $y(t)$ as a solution of the initial-value problem for the ode

$$
\left.\dot{y}(t)=\int u(x, t) s^{\prime}(x+y(t))\right) \mathrm{d} x, \quad y(0)=0,
$$

where $u(x, t)$ is a given solution. Introduce the function

$$
\Phi(t):=\frac{1}{2} \int[u(x, t)-s(x+y(t))]^{2} \mathrm{~d} x
$$

(it is easy to see that for the shift $y_{0}$ for which the integral above is minimal, $\left.\int u(x) s^{\prime}\left(x+y_{0}\right)=0\right)$.

We compute the derivative of $\Phi$ and substitute $u_{t}$ from the equation (7) to obtain

$$
\begin{aligned}
\frac{\mathrm{d}}{\mathrm{d} t} \Phi(t)= & \int(u-s)\left[-\dot{y} s^{\prime}(x+y(t))-u_{x} u+u_{x x}+\alpha u-\alpha A u\right] \\
= & -\left\|u_{x}\right\|^{2}+\alpha\|u\|^{2}-\dot{y} \int u s^{\prime}-\alpha \int u A u \\
& -\int s u_{x x}+\int s u_{x} u-\alpha \int s u+\alpha \int s A u .
\end{aligned}
$$

In the calculation above and everywhere in what follows we keep the notation $s$ for the shifted function $s(\cdot+y(t))$; we also use $s_{0}$ for the unshifted $s(\cdot+0)$. Taking into account the definition of $\dot{y}$ and $(8)$, we continue the estimate:

$$
\leqslant-\left\|u_{x}\right\|^{2}+\alpha\|u\|^{2}-\left\langle u s^{\prime}\right\rangle^{2}+\int s u_{x} u-\int s u_{x x}-\alpha \int s u+\alpha \int s A u .
$$

Terms containing $s$ are estimated as follows:

$$
\begin{aligned}
\underbrace{\ldots} & =-\frac{1}{2} \int s^{\prime} u^{2}+\int s^{\prime} u_{x}-\alpha \int(s-A s) u \\
& \leqslant-\frac{1}{2} \int s^{\prime} u^{2}+\frac{1}{2}\left\|s^{\prime}\right\|^{2}+\frac{1}{2}\left\|u_{x}\right\|^{2}+\frac{\alpha}{2}\|s-A s\|^{2}+\frac{\alpha}{2}\|u\|^{2}
\end{aligned}
$$

Then we have

$$
\begin{aligned}
\frac{\mathrm{d}}{\mathrm{d} t} \Phi(t) & =\frac{1}{2} \frac{\mathrm{d}}{\mathrm{d} t}\|u-s\|^{2} \\
& \leqslant-\left\langle u s^{\prime}\right\rangle^{2}-\frac{1}{2}\left\|u_{x}\right\|^{2}+\int\left(\frac{3}{2} \alpha-\frac{1}{2} s^{\prime}\right) u^{2}+\frac{1}{2}\left\|s^{\prime}\right\|^{2}+\frac{\alpha}{2}\|s-A s\|^{2} \\
& =-\left\langle u s^{\prime}\right\rangle^{2}-\frac{1}{2}\left\|u_{x}\right\|^{2}+\int u^{2}\left(2 \alpha-\frac{1}{2} s^{\prime}\right)-\frac{\alpha}{2}\|u\|^{2}+\frac{1}{2}\left(\left\|s^{\prime}\right\|^{2}+\alpha\|s-A s\|^{2}\right) .
\end{aligned}
$$

So far $s$ was arbitrary. Now we select $s$ that satisfies

$$
s^{\prime}=4 \alpha-2 b(x)
$$

where the particular choice of $b(x)$ is described in the lemma above. 
To guarantee $s$ being periodic, we demand

$$
\int s^{\prime}=\int(4 \alpha-2 b(x))=4 \alpha L-2 B=0,
$$

which implies $B=2 \alpha L$. Note that

$$
\int u b=\langle u b\rangle=\left\langle u\left(2 \alpha-\frac{1}{2} s^{\prime}\right)\right\rangle=-\frac{1}{2}\left\langle u s^{\prime}\right\rangle
$$

and consequently $\langle u b\rangle^{2}$ in the inequality 14 can be replaced by $\left\langle u s^{\prime}\right\rangle^{2} / 4$. Then

$$
\begin{aligned}
\frac{1}{2} \frac{\mathrm{d}}{\mathrm{d} t}\|u-s\|^{2} & \underset{L>1 /(2 \alpha)}{\underset{14}{\leqslant}}\left(-\frac{1}{4}\left\|u_{x}\right\|^{2}-\frac{\alpha}{2}\|u\|^{2}+\frac{1}{2}\left(\left\|s^{\prime}\right\|^{2}+\alpha\|s-A s\|^{2}\right)\right. \\
& \leqslant-\frac{1}{4}\left\|u_{x}\right\|^{2}-\frac{\alpha}{4}\|u-s\|^{2}+\frac{\alpha}{2}\|s\|^{2}+\frac{1}{2}\left(\left\|s^{\prime}\right\|^{2}+\alpha\|s-A s\|^{2}\right)
\end{aligned}
$$

where in the last step we used an elementary inequality

$$
-\|u\|^{2} \leqslant-\frac{1}{2}\|u-s\|^{2}+\|s\|^{2}
$$

that follows from the triangle inequality. Thus,

$$
\frac{1}{2} \frac{\mathrm{d}}{\mathrm{d} t}\|u-s\|^{2} \leqslant-\frac{\alpha}{4}\|u-s\|^{2}+\underbrace{\frac{1}{2}\left(\left\|s^{\prime}\right\|^{2}+\alpha\|s\|^{2}+\alpha\|s-A s\|^{2}\right)} .
$$

The bound for the terms $\underbrace{\ldots}$ is easily obtained. First,

$$
\frac{1}{2}\left\|s^{\prime}\right\|^{2}=-8 \alpha^{2} L+\frac{64}{3} B^{3} \underset{B=2 \alpha L}{\leqslant} C_{1}(\alpha L)^{3},
$$

and also

$$
\frac{1}{2}\left(\|s\|^{2}+\|s-A s\|^{2}\right) \leqslant \frac{1}{2}\left(2+\|A\|^{2}\right)\|s\|^{2} \underset{\|A\|<1}{<} \frac{3}{2}\|s\|^{2}:=\frac{3}{2} C_{2} \alpha^{2} L^{3}
$$

where $C_{1}$ and $C_{2}$ are absolute constants that are obtained explicitly from (18) through integration. Therefore, assuming $\alpha \geqslant \alpha_{0}>0$, we see that $\underbrace{\ldots} \leqslant C(\alpha L)^{3}$ and consequently

$$
\frac{1}{2} \frac{\mathrm{d}}{\mathrm{d} t}\|u-s\|^{2} \leqslant-\frac{\alpha}{4}\|u-s\|^{2}+C \alpha^{3} L^{3} .
$$

Finally, from the Gronwall lemma we get

$$
\|u-s\|^{2} \leqslant\left(\left\|u_{0}-s_{0}\right\|^{2}-4 C \alpha^{2} L^{3}\right) \exp \left(-\frac{\alpha}{2} t\right)+4 C \alpha^{2} L^{3},
$$


showing the exponential approach to the absorbing set. This inequality yields

$$
\begin{aligned}
\|u\| & \leqslant\|u-s\|+\|s\| \\
& \leqslant\left|\left\|u_{0}-s_{0}\right\|^{2}-4 C \alpha^{2} L^{3}\right|^{1 / 2} \exp \left(-\frac{\alpha}{4} t\right)+2 \alpha \sqrt{C} L^{3 / 2}+\alpha \sqrt{C_{2}} L^{3 / 2} \\
& =\left|\left\|u_{0}-s_{0}\right\|^{2}-4 C \alpha^{2} L^{3}\right|^{1 / 2} \exp \left(-\frac{\alpha}{4} t\right)+\alpha L^{3 / 2} C_{a}
\end{aligned}
$$

where $C_{a}=2 \sqrt{C}+\sqrt{C_{2}}$.

REMARK 3.5 With some modifications, the above proof can be repeated for any bounded operator $A$ leading to a slightly worse estimate; however, this result is irrelevant for our model.

From the proof above it is easy to derive the following

CoRollary 3.6 Define $R_{a}=C_{a} \alpha L^{3 / 2}$. Then for any ball $B_{R}=\left\{\left\|u_{0}\right\| \leqslant R\right\}$ :

(i) for all $t>0,\|u(\cdot, t)\| \leqslant R+2 R_{a}$;

(ii) there exists $t_{0}=t_{0}(R)$ so that $\|u(\cdot, t)\| \leqslant R_{a}+\varepsilon$ for any $t>t_{0}$.

Proof. The proof is based on the estimate in 21]. First we note that

$$
\begin{aligned}
\left|\left\|u_{0}-s_{0}\right\|^{2}-4 C \alpha^{2} L^{3}\right|^{1 / 2} & \leqslant\left\|u_{0}\right\|+\left\|s_{0}\right\|+2 \alpha L^{3 / 2} \sqrt{C} \\
& \leqslant R+\sqrt{C_{2}} \alpha L^{3 / 2}+2 \alpha L^{3 / 2} \sqrt{C}=R+R_{a} .
\end{aligned}
$$

Thus 21] can be replaced by

$$
\|u\| \leqslant\left(R+R_{a}\right) \exp \left(-\frac{\alpha}{4} t\right)+R_{a} \leqslant R+2 R_{a} .
$$

It also shows that $\|u(\cdot, t)\| \leqslant R_{a}+\varepsilon$, starting from some $t_{0}$.

We note that the constants in the proof above are not necessarily optimal.

\section{Compact attractor}

A compact attractor is obtained as an $\omega$-limit set of any absorbing ball $\mathcal{B}:=B_{R_{a}+\varepsilon}$. We have already proved uniform boundedness for the orbits. To demonstrate compactness via Rellich's theorem, it is sufficient to obtain a uniform bound on the derivative:

LEMMA 4.1 For any $t \geqslant 0$, and any $r>0$,

$$
\left\|u_{x}(\cdot, t+r)\right\|^{2} \leqslant\left(\alpha+\frac{1}{r}\right) R^{2} \exp \left\{(\alpha r+1) \frac{L R^{2}}{2}+2 \alpha\right\}
$$

where $R=\|u(\cdot, 0)\|+2 R_{a}$. 
Proof. First we will derive two rather standard a priori energy estimates for solutions of (7). We multiply the equation in (7) by $u$ and integrate by parts with respect to $x$ to obtain

$$
\frac{1}{2} \frac{\mathrm{d}}{\mathrm{d} t}\|u\|^{2}=-\left\|u_{x}\right\|^{2}+\alpha\|u\|^{2}-\underbrace{\alpha \int u\left(I-\partial_{x}^{2}\right)^{-1} u}_{\geqslant 0} \leqslant-\left\|u_{x}\right\|^{2}+\alpha\|u\|^{2} .
$$

Similarly, we multiply the equation by $u_{x x}$ and integrate by parts:

$$
\begin{aligned}
\frac{1}{2} \frac{\mathrm{d}}{\mathrm{d} t}\left\|u_{x}\right\|^{2} & =-\left\|u_{x x}\right\|^{2}+\alpha\left\|u_{x}\right\|^{2}-\alpha \int u_{x} A u_{x}-\int u u_{x} u_{x x} \\
& \leqslant-\left\|u_{x x}\right\|^{2}+\alpha\left\|u_{x}\right\|^{2}+\left|\int u u_{x} u_{x x}\right|
\end{aligned}
$$

The cubic term is routinely handled as follows:

$$
\begin{aligned}
\left|\int u u_{x} u_{x x}\right| & \leqslant \max |u(x)| \int\left|u_{x} u_{x x}\right| \underset{\text { Poincaré's inequality }}{\leqslant} L^{1 / 2}\left\|u_{x}\right\| \int\left|u_{x} u_{x x}\right| \\
& \leqslant\left\|u_{x x}\right\|^{2}+\frac{1}{4} L\left\|u_{x}\right\|^{4} .
\end{aligned}
$$

Therefore

$$
\frac{1}{2} \frac{\mathrm{d}}{\mathrm{d} t}\left\|u_{x}\right\|^{2} \leqslant\left\|u_{x}\right\|^{2}\left(\alpha+\frac{1}{4} L\left\|u_{x}\right\|^{2}\right) .
$$

On the other hand, we rewrite the energy estimate (22) as

$$
\left\|u_{x}\right\|^{2} \leqslant \alpha\|u\|^{2}-\frac{1}{2} \frac{\mathrm{d}}{\mathrm{d} t}\|u\|^{2}
$$

and integrate from $t$ to $t+r$ to see that

$$
\int_{t}^{t+r}\left\|u_{x}\right\|^{2} \mathrm{~d} \tau \leqslant \alpha \int_{t}^{t+r}\|u\|^{2} \mathrm{~d} \tau-\left.\frac{1}{2}\|u\|^{2}\right|_{t} ^{t+r} \leqslant \alpha r R^{2}+R^{2}
$$

where $R=\|u(\cdot, 0)\|+2 R_{a}$ is a uniform bound for $\|u\|$ (see Corollary 3.6).

Now we can apply the uniform Gronwall lemma [Temam, Lemma III.1.1] with $y=\left\|u_{x}\right\|^{2}$, $g=2 \alpha+\frac{1}{2} L\left\|u_{x}\right\|^{2}$, and $h=0$, which yields

$$
\left\|u_{x}(\cdot, t+r)\right\|^{2} \leqslant\left(\alpha+\frac{1}{r}\right) R^{2} \exp \left\{(\alpha r+1) \frac{L R^{2}}{2}+2 \alpha\right\} .
$$

From the proof above it is easy to derive the following result that we will need for the dimension estimates.

COROLlaRY 4.2 If $u(\cdot, t)$ belongs to the attractor then

$$
\begin{gathered}
\left\|u_{x}(\cdot, t)\right\|^{2} \leqslant(\alpha+1) R_{a}^{2} \exp \left\{\frac{\alpha+1}{2} R_{a}^{2} L+2 \alpha\right\}, \\
\frac{1}{t} \int_{0}^{t}\left\|u_{x}\right\|^{2} \leqslant \alpha R_{a}^{2}+\frac{1}{t} R_{a}^{2}=\left(\alpha+\frac{1}{t}\right) R_{a}^{2} .
\end{gathered}
$$


Proof. For $u(\cdot, t)$ in the attractor, $\|u\| \leqslant R_{a}$; in this case $R$ in $(26)$ is replaced by $R_{a}$. Now we select $r=1$ in (26) to obtain (27) for $t>1$. Next, similarly to the derivation of (25) above, we integrate the estimate in (24) with respect to $t$ from 0 to $t$ and obtain (28).

Now we are ready to establish the compactness result.

THEOREM 4.3 The initial value problem (7) in the class of periodic functions with zero average possesses a maximal, connected, compact attractor in $H^{0}$.

Proof. A compact attractor is obtained as an $\omega$-limit set of the absorbing ball $\mathcal{B}:=B_{R_{a}+\varepsilon}, \mathcal{A}=$ $\bigcap_{t_{0} \geqslant 0} \overline{\Omega\left(t_{0}\right) \mathcal{B}}$, where $\Omega\left(t_{0}\right) u_{0}$ is the orbit of the time evolution $S(t) u_{0}$ with the initial condition $u_{0}$, that is, $\Omega\left(t_{0}\right) u_{0}=\bigcup_{t>t_{0}} S(t) u_{0}$. For a fixed $r=r_{0}$ the estimate in 26) is uniform for $t \geqslant r_{0}$, and for all $u_{0}$ with $\left\|u_{0}\right\| \leqslant R$. It holds for any $R$, including the absorbing ball. Together with the uniform bounds on $\|u(\cdot, t)\|$ from Corollary 3.6 it allows us to use Rellich's theorem and conclude that the orbit $\Omega\left(t_{0}\right) \mathcal{B}$ is precompact in $H^{0}$. It is known (see e.g. [Temam, Theorem I.1.1]) that in this case the $\omega$-limit set of $\mathcal{B}$ is a maximal, connected, compact attractor of the flow $S$.

\section{Dimension of attractor}

In order to obtain the estimate on the Hausdorff dimension of the attractor we study evolution of the infinitesimal volume along the orbits in the attractor. We demonstrate that for sufficiently large $m$ the " $m$-dimensional volume" decays exponentially. This property combined with compactness of the attractor and differentiability of the semigroup imply that the Hausdorff dimension of the attractor is no larger than $m$. In the argument regarding the Hausdorff dimension of the attractor we follow quite closely the ideas outlined in [Temam, Chap. V].

Let $v$ be a solution of (7p) in the attractor, and $u=v+\varepsilon w$, its perturbation. Then the linearization is given by

$$
z_{t}=-v z_{x}-z v_{x}+z_{x x}+\alpha z-\alpha\left(I-\partial_{x}^{2}\right)^{-1} z=: \mathcal{L}[v] z .
$$

Since $v$ is smooth, clearly the linearized problem is well-posed in $H^{0}$.

Now we will estimate the evolution of the volume element. To this end we need to estimate the trace of the finite-dimensional projections of the generator of the linear semigroup. Let $\xi_{j}$, $j=1, \ldots, m$, be elements of $H^{0}$ and let $z_{j}$ be the solution of the linearized problem with initial data $\xi_{j}$. Then the volume element spanned by $\left\{\xi_{1}, \ldots, \xi_{m}\right\}$ evolves according to the formula

$$
\left.\left|z_{1}(t) \wedge \cdots \wedge z_{m}(t)\right|=\mid \xi_{1} \wedge \cdots \wedge \xi_{m}\right) \mid \exp \int_{0}^{t} \operatorname{Tr}\left[\mathcal{L}[v(\tau)] \circ Q_{m}(\tau)\right] \mathrm{d} \tau
$$

where $Q_{m}(\tau)$ is the projector in $H^{0}$ onto the space spanned by $\Xi(\tau)=\left\{z_{1}(\tau), \ldots, z_{m}(\tau)\right\}$.

In order to calculate the trace we choose a basis $\left\{\phi_{1}, \ldots, \phi_{m}\right\}$ in $\Xi(\tau)$ orthonormal in $H^{0}$. For the diagonal entry of the matrix of the corresponding $m$-dimensional projection we have

$$
\left\langle\mathcal{L} \phi_{j}, \phi_{j}\right\rangle=-\left\|\partial \phi_{j}\right\|^{2}+\alpha\left\|\phi_{j}\right\|^{2}+\int v \phi_{j} \partial \phi_{j}-\alpha \int \phi_{j} A \phi_{j} .
$$


The term $\int v \phi_{j} \partial \phi_{j}$ is estimated as follows:

$$
\begin{aligned}
\left|-\int v \phi_{j} \partial \phi_{j}\right| & \leqslant \sup |v| \int\left|\phi_{j}\right|\left|\partial \phi_{j}\right| \underset{\text { Schwarz }}{\leqslant} \sup |v|\left\|\phi_{j}\right\|\left\|\partial \phi_{j}\right\| \\
& \leqslant \frac{1}{\text { Young }} \frac{1}{2}\left(\sup |v|\left\|\phi_{j}\right\|\right)^{2}+\frac{1}{2}\left\|\partial \phi_{j}\right\|^{2} \underset{\text { Poincare }}{\leqslant}\left\|\frac{1}{2}\right\| \sqrt{L} v_{x}\left\|^{2}\right\| \phi_{j}\left\|^{2}+\frac{1}{2}\right\| \partial \phi_{j} \|^{2} \\
& =\frac{1}{2} L\left\|v_{x}\right\|^{2}+\frac{1}{2}\left\|\partial \phi_{j}\right\|^{2} .
\end{aligned}
$$

For the trace we get

$$
\sum_{j=1}^{m}\left\langle\mathcal{L} \phi_{j}, \phi_{j}\right\rangle \leqslant-\frac{1}{2} \sum_{j=1}^{m}\left\|\partial \phi_{j}\right\|^{2}+\alpha m+\frac{m}{2} L\left\|v_{x}\right\|^{2}
$$

Since the eigenvalues of $\partial^{2} / \partial x^{2}$ are given by $(2 \pi j / L)^{2}, j=1,2, \ldots$, it is clear that for some absolute constant $c$,

$$
\sum_{j=1}^{m}\left\|\partial \phi_{j}\right\|^{2} \geqslant c m^{3} / L^{2}
$$

As a result we get the estimate for the trace

$$
\begin{aligned}
\frac{1}{t} \int_{0}^{t} \sum_{j=1}^{m}\left\langle\mathcal{L} \phi_{j}, \phi_{j}\right\rangle \mathrm{d} \tau & \leqslant-\frac{1}{2} c m^{3} / L^{2}+\alpha m+\frac{m}{2} L\left(\frac{1}{t} \int_{0}^{t}\left\|v_{x}\right\|^{2} \mathrm{~d} \tau\right) \\
& \leqslant-\frac{1}{2} c m^{3} / L^{2}+\alpha m+c_{1} m \alpha^{3} L^{4}
\end{aligned}
$$

where $t$ in (28) is sufficiently large.

Thus, the trace becomes negative when

$$
m \sim \alpha^{3 / 2} L^{3} .
$$

REMARK 5.1 A substantially more involved argument, based on a more precise estimate of the cubic term due to Lieb and Thirring, gives a better estimate for the dimension: $m \sim c L^{29 / 18}$. However, in view of the remarks in the introduction on the connection between dynamical behavior and dimension, we present a simpler result given above.

Next, in order to utilize the trace estimate developed above we need to demonstrate that the nonlinear evolution of the volume is well approximated by its linear counterpart. This will be ensured by the differentiability of the semigroup solving the problem with respect to the initial conditions (cf. [Temam, Sec. V.3.3]).

THEOREM 5.2 Let $U$ and $W$ be two solutions in the attractor, $U=S(t) U_{0}, W=S(t) W_{0}$, and $z(t)$ be a solution of the linearized problem 29] with the initial condition $z(0)=U_{0}-W_{0}$. Then for any $t$ with $0 \leqslant t \leqslant t_{0}$,

$$
\|U(t)-W(t)-z(t)\| \leqslant C\left\|U_{0}-W_{0}\right\|^{2}
$$

where the constant $C$ depends only on $t_{0}$. 
In this case the mapping $z(0) \mapsto z(t)$ is the Fréchet differential of $S(t)$ at the point $U_{0}$.

Proof. The difference $w=U-W$ solves the following problem:

$$
\begin{aligned}
& w_{t}=w_{x x}+\alpha w-\alpha A w-w U_{x}-W w_{x}, \\
& w(x, 0)=U_{0}(x)-W_{0}(x) .
\end{aligned}
$$

As usual we perform an energy estimate:

$$
\begin{aligned}
\frac{1}{2} \frac{\mathrm{d}}{\mathrm{d} t}\|w\|^{2} & =-\left\|w_{x}\right\|^{2}+\alpha\|w\|^{2}-\underbrace{\alpha \int w A w}_{\geqslant 0}+\int(2 U-W) w_{x} w \\
& \leqslant-\left\|w_{x}\right\|^{2}+\alpha\|w\|^{2}+\underbrace{\sqrt{L}\left(2\left\|U_{x}\right\|+\left\|W_{x}\right\|\right)}_{\text {Poincaré }}\left(\frac{1}{2 b}\|w\|^{2}+\frac{b}{2}\left\|w_{x}\right\|^{2}\right) \\
& \leqslant-\left\|w_{x}\right\|^{2}+\alpha\|w\|^{2}+3 C \sqrt{L}\left(\frac{1}{2 b}\|w\|^{2}+\frac{b}{2}\left\|w_{x}\right\|^{2}\right) \\
& \leqslant-\frac{1}{2}\left\|w_{x}\right\|^{2}+(\alpha+c)\|w\|^{2},
\end{aligned}
$$

where $C$ is the uniform estimate on $\left\|U_{x}\right\|,\left\|W_{x}\right\|$ (cf. (27)); the constant $b$ is selected so that $3 b C \sqrt{L}=1$. Therefore

$$
\|w(\cdot, t)\|^{2} \leqslant\|w(\cdot, 0)\|^{2} \exp (2(\alpha+c) t)
$$

In addition

$$
\begin{aligned}
\int_{0}^{t}\left\|w_{x}\right\|^{2} & \leqslant(\alpha+c) \int_{0}^{t}\|w\|^{2}+\|w(\cdot, t)\|^{2}-\|w(\cdot, 0)\|^{2} \\
& \leqslant\|w(\cdot, 0)\|^{2}\left[\frac{\exp (2(\alpha+c) t)-1}{2(\alpha+c)}+\exp (2(\alpha+c) t)-1\right] \\
& =: C_{0}\left\|U_{0}-W_{0}\right\|^{2} .
\end{aligned}
$$

For the difference $y=w-z$ we have the following problem:

$$
y_{t}=y_{x x}+\alpha y-\alpha A y-U_{x} y-U y_{x}+w w_{x} .
$$

We multiply the equation in 32 by $y$ and integrate by parts to obtain the following identity for the norm:

$$
\frac{1}{2} \frac{\mathrm{d}}{\mathrm{d} t}\|y\|^{2}=-\left\|y_{x}\right\|^{2}+\alpha\|y\|^{2}-\alpha \int y A y+\int U y y_{x}+\int w w_{x} y .
$$

On the right hand side we drop the positive term $\alpha \int y A y$ and use

$$
\int w w_{x} y \underset{\text { Poincaré }}{\leqslant} \sqrt{L}\left\|w_{x}\right\| \int w_{x} y \underset{\text { Schwarz }}{\leqslant} \sqrt{L}\left\|w_{x}\right\|^{2}\|y\|
$$

to estimate the right hand side of 33 as follows:

$$
\leqslant-\left\|y_{x}\right\|^{2}+\alpha\|y\|^{2}+\sqrt{L}\left\|U_{x}\right\|\left(\frac{1}{2 b}\|y\|^{2}+\frac{b}{2}\left\|y_{x}\right\|^{2}\right)+\sqrt{L}\left\|w_{x}\right\|^{2}\|y\|,
$$


We select $b$ so that

$$
\sqrt{L}\left\|U_{x}\right\| \frac{b}{2}<1
$$

which yields

or

$$
\frac{1}{2} \frac{\mathrm{d}}{\mathrm{d} t}\|y\|^{2} \leqslant C\|y\|^{2}+\sqrt{L}\left\|w_{x}\right\|^{2}\|y\|
$$

$$
\frac{\mathrm{d}}{\mathrm{d} t}\|y\| \leqslant C\|y\|+\sqrt{L}\left\|w_{x}\right\|^{2}
$$

Now from the Gronwall lemma we obtain

$$
\|y(\cdot, t)\| \leqslant e^{C t} \int_{0}^{t} \sqrt{L}\left\|w_{x}\right\|^{2} e^{-C \tau} \mathrm{d} \tau \underset{[31}{\leqslant} \sqrt{L} e^{C t} C_{0}\left\|U_{0}-W_{0}\right\|^{2},
$$

thus completing the proof of Theorem 5.2

Finally, based on [Temam, Theorem V.3.1], the dimension estimate [30] and the differentiability Theorem 5.2 allow us to obtain

THEOREM 5.3 The Hausdorff dimension of the attractor for the problem (7) in the class of periodic functions with zero average is finite,

$$
\operatorname{dim}_{H}(\mathcal{A}) \sim \alpha^{3 / 2} L^{3} .
$$

\section{Acknowledgments}

The work of C.M.B. and J.H. was supported in part by the RTN network "Nonlinear Partial Differential Equations describing Front Propagation and other Singular Phenomena", HPRNCT-2002-00274. M.F. was supported in part by the National Science Foundation, Grant DMS0207308, and CNRS. The warm hospitality of the Vrije Universiteit Amsterdam and the Université Bordeaux 1 is gratefully acknowledged. The authors would like to express their gratitude to the anonymous referee for the thoughtful and detailed remarks and suggestions.

\section{REFERENCES}

[BKS01] Berestycki, H., KAmin, S., \& Sivashinsky, G. Metastability in flame front evolution. Interfaces Free Bound. 3 (2001), 361-392. Zb1 0991.35097 MR 1869585

[BFHS05] Brauner, C.-M, Frankel, M., Hulshof, J., \& Sivashinsky, G. I. Weakly nonlinear asymptotics of the $\kappa-\theta$ model of cellular flames: the QS equation. Interfaces Free Bound. 7 (2005), 131-146. Zbl pre02201121 MR 2148913

[BL00] BRAUner, C.-M., \& LunARDI, A. Instabilities in a two-dimensional combustion model with free boundary. Arch. Ration. Mech. Anal. 154 (2000), 157-182. Zbl 0984.76030 MR 1784964

[CEeS93] Collet, P., Eckmann, J.-P., Epstein, H., \& Stubbe, J. A global attracting set for the Kuramoto-Sivashinsky equation. Comm. Math. Phys. 152 (1993), 203-214. Zbl 0777.35073 MR 1207676

[FGS03] Frankel, M. L., Gordon, P. V., \& Sivashinsky, G. I. On disintegration of near-limit cellular flames. Phys. Lett. A 310 (2003), 389-392. MR 1976154

[GA04] Giacomelli, L., \& Otтo, F. New bounds for the Kuramoto-Sivashinsky equation. Comm. Pure Appl. Math. 58 (2005), 297-318. Zbl 1062.35113 MR 2116616 
[G94] Goodman, J. Stability of the Kuramoto-Sivashinsky and related systems, Comm. Pure Appl. Math. 47 (1994), 293-306. Zbl 0809.35105 MR 1266243

[MS79] Matkowsky, B. J., \& Sivashinsky, G. I. On asymptotic derivation of two models in flame theory associated with the constant density approximation. SIAM J. Appl. Math. 37 (1979), 686699. Zbl 0437.76065 MR 0549149

[NST85] Nicolaenko, B., Scheurer, B., \& Temam, R. Some global dynamical properties of the Kuramoto-Sivashinsky equations: nonlinear stability and attractors. Phys. D 16 (1985), 155-183. Zbl 0592.35013 MR 0796268

[RS87] RAKIB, Z., \& SivASHINSKY, G. I. Instabilities in upward propagating flames. Combust. Sci. Technol. 54 (1987) 69-84.

[Siv] Sivashinsky, G. I. Nonlinear analysis of hydrodynamic instability of in laminar flames, Part I. Derivation of basic equations. Acta Astronaut. 4 (1977), 1177-1206. Zbl 0427.76047 MR 0502829

[Temam] Temam, R. Infinite-Dimensional Dynamical Systems in Mechanics and Physics. 2nd ed., Appl. Mat. Sci. 68, Springer (1997). Zbl 0871.35001 